\title{
Exploring Social Context and Psychological Distress in Adult Canadians with Cannabis Use Disorder: To What Extent Do Social Isolation and Negative Relationships Predict Mental Health?
}

\author{
Tracy L. Gulliver ${ }^{1}$ (D) $\cdot$ Ken Fowler $^{2}$ (D)
}

Accepted: 11 September 2021 / Published online: 28 September 2021

(C) The Author(s), under exclusive licence to Springer Science+Business Media, LLC, part of Springer Nature 2021

\begin{abstract}
The objectives of this study were to explore perceived social support, negative social interactions, and psychological distress in Canadian adults who experience lifetime abuse, or dependence on cannabis (ADC), and to determine whether, and the extent to which variables of interest predict psychological distress. Data were extracted from a cross-sectional, national datafile representing a sample of 1503 individuals who met the criteria for a lifetime prevalence of ADC. Levels of perceived overall social support, and several subtypes were measured using the Social Provisions Scale (SPS), negative social interactions were assessed using the Negative Social Interaction (NSI) scale, and psychological distress was examined using the Kessler Psychological Distress Scale (K10). It was observed that Canadians with ADC had significantly lower SPS scores (overall, and by subtype) and significantly higher NSI and K10 scores compared with the overall Canadian adult sample. An exploratory stepwise regression revealed that NCI scores were the most significant, positive predictor of psychological distress, which alone accounted for 20 percent of the variance, followed by reassurance of worth, attachment, and social integration which were inversely related to psychological distress. With the recent legalization of cannabis in Canada, the results of this study suggest that abuse may strongly link with negative social consequences that might serve to exacerbate psychological distress. As such, it might be beneficial to clearly understand one's social context when considering medicinal purposes of cannabis for mental health symptom management. Further, the findings also suggest that patients with cannabis addiction will likely benefit from receiving particular forms of social support. Limitations of this study and future research are considered.
\end{abstract}

Keywords Cannabis use disorder $\cdot$ Social support $\cdot$ Psychological distress $\cdot$ Negative relationships $\cdot$ Canadian adults

Cannabis is one of the most widely used psychoactive substances in Canada. According to a recent National Cannabis Survey, 27\% of Canadians aged 15 and older reported cannabis use in the previous twelve months, and $18 \%$ indicated they used cannabis daily [1]. The rate

Tracy L. Gulliver

tgulliver@mun.ca

Extended author information available on the last page of the article 
of cannabis use in Canada has risen in recent years, and following legalization in October 2018, national surveys also indicate an increased social acceptability of the drug [2]. Furthermore, $22 \%$ of Canadians have reported more frequent use of cannabis in response to the COVID-19 pandemic and stay-at-home orders [1].

In addition to recreational use, cannabis is also prescribed for medical purposes. Under the Canadian federal cannabis program, two categories of individuals are authorized to possess cannabis for medical use: those diagnosed with multiple sclerosis, spinal cord injury or disease, cancer, HIV/AIDS, epilepsy, or arthritis, and those with other symptoms assessed by a physician or a specialist [3]. In recent years, the number of Canadians who have federally authorized access to medical cannabis has also increased. For instance, as of December 2020, there were more than 320,000 medical cannabis clients registered in Canada, up from just 24,000 that were registered as of June 2015 [1].

The most common reason individuals seek out medical cannabis is for the treatment of chronic pain, and while it has been proposed that it may have salutary benefits in the treatment of mental health issues, such as managing distress and anxiety, this has not been proven in the literature [4]. Moreover, some have proposed that it is currently premature to recommend cannabis-based interventions for mental health [5]. Such perspectives are supported by the fact that frequent cannabis use (both medically and recreationally) tends to be associated with a number of wellness risks, including the potential for addiction, higher levels of psychological distress, and lower perceived levels of perceived social support [6-8].

\section{Cannabis Use Disorder}

The widespread use of both medical and recreational cannabis has led to concerns over the potential for addiction. Volkow and colleagues reported that approximately 1 in 10 adults who use cannabis will become addicted, and the risk increases to 1 in 6 for those who start using cannabis as a teen [4]. The highest risk falls to those who use cannabis daily - between $1 / 4$ and $1 / 2$ of those users will develop an addiction. The risk of cannabis addiction may also be higher for those with a pre-existing mental health condition such as depression or anxiety $[9,10]$.

According to the DSM-5, cannabis use disorder (CUD) is described as a problematic pattern of cannabis use that leads to significant impairment or distress [11]. Common symptoms include cravings, interpersonal problems, failure to fulfill work or school obligations, tolerance, and withdrawal. Research has shown that states with approved cannabis laws have increased rates of CUD and higher rates of treatment admissions [12]. These effects are found to be even larger in states that permit cannabis dispensaries and personal cultivation [13].

Researchers have found CUD to be associated with a number of negative mental health outcomes [14, 15]. Agosti and colleagues found that $90 \%$ of respondents with cannabis dependence had a lifetime mental disorder, compared to $55 \%$ without cannabis dependence. CUD has been associated with a wide range of mental disorders including major depression, anxiety disorders, bipolar disorder, and post-traumatic stress disorder (PTSD) $[14,15]$. Furthermore, individuals with cannabis dependence are more likely to suffer from other substance use disorders [16]. 
Frequent cannabis use has also been associated with higher levels of psychological distress [7]. Daily cannabis use was found to be significantly more common among individuals with serious psychological distress, and it has been increasing in this group [7]. Similarly, Moitra and colleagues found that the use of cannabis to cope with negative emotions is associated with increased psychological distress and cannabis-related problems; it is also correlated with a diagnosis of DSM-5 cannabis use disorder [17].

\section{Social Support}

In addition to psychological distress, individuals with CUD report lower levels of perceived social support and higher levels of social avoidance [8, 18]. Dorard and colleagues found that individuals seeking treatment for cannabis dependence were significantly more likely to report lower levels of perceived social support and demonstrate higher levels of social-avoidant and distraction-avoidant coping scores. Similarly, Gliksberg and colleagues found that increased cannabis use was associated with decreased social network size and diversity, which was in turn associated with lower levels of perceived social support [18].

Low levels of perceived social support have been associated with higher levels of psychological distress in individuals with substance use disorders [19] and mental disorders such as bipolar disorder [20]. Social support also has an effect on the severity and length of some mental disorders. For example, Wareham and colleagues found that positive social support was associated with significantly decreased duration of depression [21]. Furthermore, Rapier and colleagues found that social support has a protective effect against substance abuse. Specifically, significant negative correlations were found between social support scores and lifetime use of alcohol, tobacco, and cannabis [22].

From these studies, it is clear that frequent cannabis use can have a negative impact on social and interpersonal relationships. This, in turn, is associated with higher levels of substance abuse and increased avoidance of social interactions. Individuals with CUD and low levels of social support may be especially vulnerable to negative mental health outcomes and increased psychological distress. Cannabis may also act as a barrier to treatment in vulnerable users [23, 24]. Another variable of interest that has not been frequently discussed in the literature is negative social interactions. It is possible that individuals with CUD may avoid social interactions because the majority of these interactions are negative; this may further exacerbate psychological distress.

\section{Current Study}

The goals of the current study are to (1) investigate the rate of Canadians who experience lifetime abuse, or dependence on cannabis (ADC) (2) assess levels of perceived social support (overall and subtypes) and psychological distress in the ADC sample compared to the overall CCHS-MH sample, and (3) determine whether, and the degree to which age, sex, income, the subtypes of social support, and negative social interactions predict psychological distress in the ADC sample. 


\section{Method}

\section{Participants}

Data from the Public Use Microdata File of the Canadian Community Health SurveyMental Health (CCHS-MH) 2012 [25] were analyzed. The CCHS-MH interviewed individuals aged 15 and older within the 10 provinces of Canada. To achieve a representative sample of the Canadian population, participants were randomly selected from an area frame, a list frame of telephone numbers as well as a random digit dialling frame. The national response rate for the survey was $68.9 \%$ resulting in data from 25,113 Canadians [25]. The present study included adults aged 20-64 years. Within this sample, 1503 Canadians met the criteria for lifetime abuse or dependence on cannabis.

\section{Data Collection}

Data were collected from January 1, 2012 to December 31, 2012. A large portion (87\%) of the interviews were conducted in person and the remainder were conducted by telephone. The survey was administered using the Computer-Assisted Personal Interviewing (CAPI) method which asks additional questions based on participant responses. The survey was delivered by trained representatives from Statistics Canada [25].

\section{Materials}

Cannabis Abuse or Dependence: Lifetime Prevalence The rate of Canadians who experienced lifetime abuse or dependence on cannabis (ADC) was determined using the "Cannabis Abuse or Dependence - Lifetime" algorithm. The questions in this algorithm were based on the World Health Organization version of the Composite International Diagnostic Interview (WHO-CIDI), modified for the needs of CCHS-MH [25]. The WHO-CIDI is a comprehensive, fully structured interview designed to be delivered by trained interviewers [26]. It used criteria from the DSM-IV to determine the diagnosis of a mental disorder. If a respondent met the criteria for ADC, it indicates that they experienced abuse or dependence on cannabis at least once in their lifetime.

The Kessler Psychological Distress Scale (K10) Psychological distress in participants was assessed using the K10, a 10-item questionnaire used to assess anxiety and mood over the past 4 weeks [25]. An example of a question from the K10 is "During the past month, how often did you feel worthless?". Participants rate each question on a scale of 0 (none of the time) to 4 (all of the time). The scores from the 10 questions are summed to create a total score, which can range from 0 to 40 . Low scores indicate low levels of psychological distress, and high scores indicate high levels of psychological distress.

The Social Provisions Scale (SPS) Social support in participants was assessed using the SPS. This 10-item questionnaire is used to assess current relationships with friends, family members, co-workers, community members, etc. The SPS consists of 5 social support subtypes that combine to give an overall score [25]. The social support subtypes are attachment (i.e., emotional closeness), guidance (i.e., advice or information), reliable alliance (i.e., assurance that others can be counted on in times of stress), social integration (i.e., 
sense of belonging to a group of friends), and reassurance of worth (i.e., recognition of one's competence). Responses to items within this scale were rated on a 4-point scale ranging from 1 (strongly agree) to 4 (strongly disagree). Overall scores on the SPS range from 10-40, with higher scores reflecting a higher level of perceived social support.

Negative Social Interaction Negative social interactions in participants were assessed using the Negative Social Interaction (NSI) scale. This is a 5-item questionnaire used to assess negative interactions with others over the past 4 weeks [25]. An example of a question from the NSI is "During the past month, how often have you felt that others acted angry or upset with you?". Participants rate each question on a scale of 0 (never) to 4 (very often). Low scores indicate low levels of negative social interaction, and high scores indicate high levels of negative social interaction.

\section{Statistical Analysis}

A one-sample $t$ test was used to compare the K10 score means between participants with ADC and the overall CCHS-MH sample. A series of one-sample t tests were used to compare the SPS score means and subtypes of SPS score means between participants with ADC and the overall CCHS-MH sample. Finally, a stepwise regression was conducted to examine whether and the extent to which age, sex, personal income, the subtypes of social support, and negative social interactions predicted psychological distress in the ADC sample.

\section{Results}

Demographics of the lifetime abuse or dependence on cannabis (ADC) sample are shown in Table 1. Males were much more likely to meet the criteria for ADC than females $(69.1 \%$ vs. $30.9 \%$, respectively). In terms of age, the majority of the ADC sample were under 44 years of age $(64.6 \%)$. Individuals in the 20 to 24 years age group were most likely to meet the criteria for ADC (17.8\%). Furthermore, only $26.9 \%$ of the sample reported being married, and a total of $55.6 \%$ were single, divorced, separated, or widowed.

A one-sample $t$ test revealed that psychological distress (K10) scores in the sample of adults with $\mathrm{ADC}(n=1503, M=8.34, S D=6.83)$ were significantly higher than the overall CCHS-MH sample $(n=16,972, M=5.79, S D=5.81), t(1501)=14.47, p<.001, d=0.37$, $95 \%$ CI $[2.20,2.90]$. This indicates a medium effect. These results are shown in Table 2. A one-sample $t$ test also revealed that the ADC sample were significantly lower in overall SPS scores measuring social support than the CCHS-MH sample, $t(1473)=-3.77, p<.001$, $d=0.10,95 \%$ CI $[-0.71,-0.23]$, indicating a small effect.

A series of one-sample t tests revealed that the ADC sample scored significantly lower in all subtypes of social support compared to the overall sample. Specifically, adults with ADC scored significantly lower in attachment $t(1492)=-2.46, p<.05, d=0.06,95 \%$ CI $[-0.13,-0.01]$; guidance $t(1495)=-2.27, p<.05, d=0.06,95 \%$ CI $[-0.12,-0.01]$; reliable alliance $t(1495)=-2.70, p<.05, d=0.07,95 \%$ CI $[-0.13,-0.02]$; social integration $t(1488)=-4.80, p<.001, d=0.12,95 \%$ CI [-0.21, -0.09]; and reassurance of worth $t(1484)=-3.53, p<.001, d=0.09,95 \%$ CI $[-0.16,-0.05]$. All effect sizes were small. A final one-sample t test revealed that the ADC sample had significantly more negative social 
Table 1 Demographics of the ADC Sample

\begin{tabular}{llr}
\hline Demographic Variable & $N$ & $\%$ \\
\hline Sex & & \\
Male & 1038 & 69.1 \\
Female & 465 & 30.9 \\
Age & & \\
20 to 24 years & 267 & 17.8 \\
25 to 29 years & 209 & 13.9 \\
30 to 34 years & 217 & 14.4 \\
35 to 39 years & 140 & 9.3 \\
40 to 44 years & 138 & 9.2 \\
45 to 49 years & 148 & 9.8 \\
50 to 54 years & 172 & 11.4 \\
55 to 59 years & 140 & 9.3 \\
60 to 64 years & 72 & 4.8 \\
Marital Status & & \\
Married & 404 & 26.9 \\
Common law & 260 & 17.3 \\
Widowed, separated, or divorced & 199 & 13.2 \\
Single & 638 & 42.4 \\
Not stated & 2 & 0.1 \\
\hline$N=1503$ & & \\
\hline
\end{tabular}

interactions than the overall sample $(t(1495)=14.46, p<.001, d=0.37,95 \%$ CI $[0.80$, 1.05]), indicating a medium effect. These results can also be seen in Table 2.

Next, a stepwise multiple regression analysis was conducted to assess the extent to which age, sex, personal income, the subtypes of social support, and negative social interactions predicted psychological distress in the ADC sample. A stepwise regression was chosen because the regression was exploratory in nature, with the goal of identifying the

Table 2 Means, Standard Deviations, t-values, df, and Cohen's d associated with Psychological Distress, SPS-10 Subscales, and Negative Social Interactions for the Entire CCHS-MH Sample and the ADC Sample

\begin{tabular}{|c|c|c|c|c|c|c|c|}
\hline & \multicolumn{2}{|c|}{$\begin{array}{l}\text { Entire CCHS-MH } \\
\text { Sample } \\
N=16,972\end{array}$} & \multicolumn{2}{|c|}{$\begin{array}{l}\text { ADC Sample } \\
N=1503\end{array}$} & \multirow[b]{2}{*}{$t$} & \multirow[b]{2}{*}{$d f$} & \multirow[b]{2}{*}{ Cohen's $d$} \\
\hline & $M$ & $S D$ & $M$ & $S D$ & & & \\
\hline Psychological Distress & 5.79 & 5.81 & 8.34 & 6.83 & $14.47 * *$ & 1501 & 0.37 \\
\hline Social Provisions Scale & 36.01 & 4.43 & 35.54 & 4.78 & $-3.77 * *$ & 1473 & 0.10 \\
\hline Attachment & 7.25 & 1.01 & 7.18 & 1.10 & $-2.46^{*}$ & 1492 & 0.06 \\
\hline Guidance & 7.32 & 1.02 & 7.26 & 1.08 & $-2.27 *$ & 1495 & 0.06 \\
\hline Reliable alliance & 7.37 & 0.94 & 7.30 & 1.04 & $-2.70 *$ & 1495 & 0.07 \\
\hline Social integration & 6.99 & 1.11 & 6.84 & 1.20 & $-4.80 * *$ & 1488 & 0.12 \\
\hline Reassurance of worth & 7.02 & 1.03 & 6.92 & 1.11 & $-3.53 * *$ & 1484 & 0.09 \\
\hline Negative Social Interactions & 2.93 & 2.32 & 3.86 & 2.48 & $14.46 * *$ & 1495 & 0.37 \\
\hline
\end{tabular}

$* p<0.05 ; * * p<0.001$ 
strongest predictors of psychological distress. The resulting model includes six significant predictors: negative social interaction, reassurance of worth, total personal income, attachment, age, and social integration. These results can be seen in Table 3.

Step 1 of the analysis reveals that the most significant predictor of psychological distress was negative social interaction, $F(1,1404)=351.19, p<.001, R^{2}=0.20$. Higher levels of negative social interaction predicted higher levels of psychological distress. Step 2 of the analysis revealed that reassurance of worth negatively predicted psychological distress, $F(2,1404)=307.73, p<.001, R^{2}=0.31$. Higher levels of reassurance of worth predicted lower levels of psychological distress.

Step 3 of the analysis revealed that total personal income negatively predicted levels of psychological distress, $F(3,1404)=243.01 p<.001, R^{2}=0.34$. Lower levels of personal income predicted higher levels of psychological distress. Step 4 revealed that attachment negatively predicted psychological distress, $F(4,1404)=197.44 p<.001$, $R^{2}=0.361$. Higher levels of attachment predicted lower levels of psychological distress. Step 5 revealed that age negatively predicted psychological distress, $F(5,1404)=159.30$

Table 3 Summary of Stepwise Regression Analysis of Variables Predicting Psychological Distress in the ADC sample

\begin{tabular}{|c|c|c|c|c|}
\hline Variable & $B$ & $S E$ & $R$ & $R^{2}$ \\
\hline \multicolumn{5}{|l|}{ Step 1} \\
\hline Negative social interaction & 1.21 & 0.07 & $0.45^{*}$ & 0.20 \\
\hline \multicolumn{5}{|l|}{ Step 2} \\
\hline Negative social interaction & 1.04 & 0.06 & & \\
\hline SPS-10: Reassurance of worth & -2.01 & 0.14 & $0.55 *$ & 0.31 \\
\hline \multicolumn{5}{|l|}{ Step 3} \\
\hline Negative social interaction & 1.02 & 0.06 & & \\
\hline SPS-10: Reassurance of worth & -1.77 & 0.14 & & \\
\hline Total personal income & -0.83 & 0.09 & $0.59 *$ & 0.34 \\
\hline \multicolumn{5}{|l|}{ Step 4} \\
\hline Negative social interaction & 0.98 & 0.06 & & \\
\hline SPS-10: Reassurance of worth & -1.05 & 0.18 & & \\
\hline Total personal income & -0.82 & 0.09 & & \\
\hline SPS-10: attachment & -1.12 & 0.18 & $0.601 *$ & 0.361 \\
\hline \multicolumn{5}{|l|}{ Step 5} \\
\hline Negative social interaction & 0.96 & 0.06 & & \\
\hline SPS-10: Reassurance of worth & -1.07 & 0.18 & & \\
\hline Total personal income & -0.77 & 0.10 & & \\
\hline SPS-10: attachment & -1.16 & 0.18 & & \\
\hline Age & -0.13 & 0.06 & $0.602^{*}$ & 0.363 \\
\hline \multicolumn{5}{|l|}{ Step 6} \\
\hline Negative social interaction & 0.94 & 0.06 & & \\
\hline SPS-10: Reassurance of worth & -0.91 & 0.19 & & \\
\hline Total personal income & -0.76 & 0.10 & & \\
\hline SPS-10: attachment & -0.95 & 0.20 & & \\
\hline Age & -0.14 & 0.06 & & \\
\hline SPS-10: social integration & -0.42 & 0.19 & $0.604 *$ & 0.365 \\
\hline
\end{tabular}


$p<.001, R^{2}=0.363$. Higher age predicted lower levels of psychological distress. Finally, step 6 revealed that social integration negatively predicted psychological distress, $F(6$, $1404)=134.02 p<.001, R^{2}=0.365$. Higher levels of social integration predicted lower levels of psychological distress. These results can also be seen in Table 3.

\section{Discussion}

Consistent with previous research, males were significantly more likely than females to meet the criteria for lifetime prevalence of cannabis abuse or dependence, an observation widely reported in the literature (e.g., [27]). Possible reasons for this include women's differing perceptions of cannabis-related harms and a greater vulnerability to the acute effects of cannabis. For instance, females reportedly see a greater risk in the regular use of cannabis [28], and recent research has shown that females may experience acute effects of cannabis at a lower dose compared to males [29].

The results of this study also revealed that most of the ADC sample were younger individuals under 44 years of age, a finding consistent with research such as the 2020 National Cannabis Survey whereby younger Canadians are significantly more likely to report cannabis use than older Canadians [1]. Specifically, youth between the ages of 20 and 24 report using cannabis over twice as much as those aged 25 and older (52\% vs. $24 \%$, respectively). However, very current research has shown increases in cannabis use among adults after 2007, indicating that this trend may be decreasing [30]. Finally, individuals in the ADC sample were more likely to be single, divorced, separated, or widowed. This reflected the findings of national data surveys in other countries such as in the United Kingdom in that frequent cannabis users are significantly more likely to report not being married or cohabiting, and having no dependent children [31].

One of the goals of this study was to identify whether Canadians with lifetime cannabis abuse or dependence (ADC) differ in terms of their perceived social support from what would be expected within the general Canadian adult population. Results indicated that Canadians with ADC perceive significantly lower levels of social support than the overall Canadian sample, an observation consistent with previous research that found cannabis use disorder (CUD) to be associated with lower levels of perceived social support [18] and reduced social network size and diversity [8]. Adults with ADC also scored significantly lower in specific social support subtypes, including attachment (i.e., they were less likely to feel they had someone they were close with), guidance (i.e., they were less likely to feel they had someone to help with making decisions and provide advice), reliable alliance (i.e., they were less likely to feel that others can be counted on in times of stress), social integration (i.e., they were less likely to feel a sense of belonging to a group), and reassurance of worth (i.e., they were less likely to feel recognized by others for their competence). Adults with ADC were also significantly more likely to experience negative social interactions than the general Canadian adult sample, suggesting that individuals with ADC experience fewer and lower quality social interactions than the general population.

Another aim of this study was to assess whether Canadians with ADC differ in terms of their psychological distress compared with the overall Canadian adult sample. Consistent with previous research, Canadians with ADC did report significantly higher levels of psychological distress [7, 17]. This could be due to the association of cannabis use disorder with higher levels of depression, anxiety, and other mental disorders [14], leading to increased levels of psychological distress. 
Unique to this study, a final goal was to determine whether, and the extent to which particular variables (i.e., age, sex, income, social support subtypes, and NSI) predicted psychological distress in the ADC sample. Results indicated that the most significant predictor of psychological distress was negative social interaction (NSI) score, accounting for $20 \%$ of the variance. Given that we also found less perceived social support in the ADC sample, it is possible that these individuals may avoid social interactions because the majority are negative, leading to further increases in psychological distress.

The second most significant predictor of psychological distress was reassurance of worth, contributing to a total of $31 \%$ of the variance in psychological distress scores. If individuals who abuse or are dependent on cannabis feel recognized by others for their competence, they are less likely to experience psychological distress. Therefore, reassurance of worth may act as a protective effect against psychological distress in cannabis users. The next most significant predictor of psychological distress was total personal income, with lower income associated with higher levels of distress.

Other significant predictors of psychological distress were attachment, age, and social integration, contributing to a total of $36.5 \%$ of the variance in psychological distress scores. Consistent with previous research, younger individuals are more likely to abuse or become dependent on cannabis [1], which may lead to higher levels of psychological distress. Furthermore, if individuals in the ADC sample felt that they had someone they were close with (i.e., attachment) or if they felt a sense of belonging to a group of friends (i.e., social integration) they were less likely to experience psychological distress. Therefore, attachment and social integration may also act as protective factors in individuals that abuse cannabis.

The results of this study indicate that heavy cannabis users are likely to suffer in terms of mental wellness and social connection, and such findings have important implications for considering cannabis-based treatments for mental health. Due to the lack of research supporting such interventions [5], and the risks to mental health from frequent use, it seems that medical cannabis may have unintended social consequences and cause more harm than benefit. Further, with the recent legalization of cannabis in Canada, the results of this study suggest that since abuse and negative social outcomes are highly associated, this may help account for the notably and consistently high psychological distress evident in this group. Consequently, there is ample justification to clearly delineate the extent and quality of one's social context when considering medicinal cannabis for mental health symptom management.

\section{Limitations}

Despite the significant results of this study, there are some limitations that need to be addressed. The main limitation is that this is a correlational study, therefore we cannot definitively say what causes cannabis abuse. Our knowledge of the directionality of the relationship is limited as well; we do not know whether the variables of interest are causing the cannabis abuse or vice versa. It is also possible that an unknown third variable is confounding our results. Another limitation of this study is that all data was collected through a survey. This type of data collection can result in self-reporting bias. In addition, certain questions run the risk of being interpreted differently by different people (i.e., psychological distress may be classified differently for different subjects). Although this type of research does yield important information, controlled (experimental) or longitudinal research is required to gain further insight. 
Another limitation is the age of the data set. Specific questions from the WHO-CIDI that determine cannabis abuse/dependence were only asked in the Mental Health version of the CCHS, which was administered in 2012 and 2019. At the time of writing (Spring 2021), the 2019 data set has not yet been released. Future research should analyze the 2019 version of the survey to assess any changes that may have occurred following legalization in October 2018. In fact, this study could be used as baseline data to compare levels of perceived social support and psychological distress pre and post legalization. Future research could also examine potential gender differences in the ADC sample.

\section{Conclusion}

Bearing the limitations in mind, this study provides valuable information for individuals considering cannabis-based medical interventions, and those suffering from cannabis addiction. Canadians who use cannabis heavily report lower levels of perceived social support and experience higher levels psychological distress than the general population of Canadians. The most significant predictor of psychological distress in the ADC sample were negative social interactions. Reassurance of worth, attachment, and social integration served as protective factors against psychological distress in the ADC sample. Finally, age and income were inversely associated with psychological distress.

The results of this study indicate that medical cannabis interventions may have unintended social consequences, and this should be considered if prescribing cannabis for mental health treatment. The results also indicate that patients suffering from cannabis addiction will likely benefit from receiving additional social support, specifically in the forms of reassurance of worth, attachment, and social integration. In addition, knowledge of at-risk groups (i.e., males with low social support) can be used in the development of prevention programs. In conclusion, cannabis use disorder is a complex disorder that is affected by many variables. In order to effectively treat cannabis abuse and dependence, the underlying factors that contribute to the addiction must be addressed.

Funding Social Sciences and Humanities Research Council of Canada funding received by Tracy Gulliver on April 16, 2020 (Canada Graduate Scholarship - Master's Program).

Availability of Data and Material (Data Transparency) All data were extracted from the public use microdata file of the Canadian Community Health Survey - Mental Health (CCHS-MH) 2012.

\section{Declarations}

Disclosure of Interest We attest that this manuscript has not been submitted elsewhere for consideration, nor is there a conflict of interest in the conduct or reporting of this research, and given data are from the Canadian Community Health Survey (CCHS) - Mental Health Cycle (2012), a public use micro file, ethics approval was not applicable for this research.

Ethics Approval Given data are from the Canadian Community Health Survey (CCHS) - Mental Health Cycle (2012), a public use microdata file, ethics approval was not applicable for this research.

Consent to Participate Not applicable.

Consent for Publication Not applicable.

Conflicts of Interest/Competing Interests Not applicable. 


\section{References}

1. Government of Canada. Cannabis Survey 2020: Summary. 2020. Retrieved from: https://www.canada. $\mathrm{ca} / \mathrm{en} /$ health-canada/services/drugs-medication/cannabis/research-data/canadian-cannabis-survey2020-summary.html.

2. Statistics Canada. What has changed since cannabis was legalized? Statistics Canada, Ottawa. 2020. Retrieved from: https://www150.statcan.gc.ca/n1/pub/82-003-x/2020002/article/00002-eng.htm.

3. Government of Canada. Medical use of cannabis. Health Canada, Ottawa. 2018. Retrieved from: https://www.canada.ca/en/health-canada/topics/cannabis-for-medical-purposes.html.

4. Watson J. The cloudy role of cannabis as a neuropsychiatric treatment. Medscape Med News. 2021. Retrieved from: https://www.medscape.com/viewarticle/950195.

5. Sarris J, Sinclair J, Karamacoska D, Davidson M, Firth J. Medicinal cannabis for psychiatric disorders: a clinically-focused systematic review. BMC Psychiatry. 2020;20(1):24. https://doi.org/10.1186/ s12888-019-2409-8.

6. Volkow ND, Baler RD, Compton WM, Weiss SR. Adverse health effects of marijuana use. N Engl J Med. 2014;370(23):2219-27. https://doi.org/10.1056/NEJMra1402309.

7. Weinberger AH, Pacek LR, Sheffer CE, Budney AJ, Lee J, Goodwin RD. Serious psychological distress and daily cannabis use, 2008 to 2016: Potential implications for mental health? Drug Alcohol Depend. 2019;197:134-40. https://doi.org/10.1016/j.drugalcdep.2019.01.010.

8. Gliksberg O, Livne O, Lev-Ran S, Rehm J, Hasson-Ohayon I, Feingold D. The association between cannabis use and perceived social support: The mediating role of decreased social network. Int J Ment Heal Addict. 2021. https://doi.org/10.1007/s11469-021-00549-4.

9. Konefal S, Gabrys R, Porath A. Clearing the smoke on cannabis: Regular use and mental health. Ottawa: Canadian Center on Substance Abuse and Addiction. 2019.

10. Buckner JD, Heimberg RG, Schneier FR, Liu S-M, Wang S, Blanco C. The relationship between cannabis use disorders and social anxiety disorder in the National Epidemiological Study of Alcohol and Related Conditions (NESARC). Drug Alcohol Depend. 2012;124(1-2):128-34. https://doi.org/10. 1016/j.drugalcdep.2011.12.023.

11. American Psychiatric Association. Diagnostic and statistical manual of mental disorders (5th ed.). 2013. https://doi.org/10.1176/appi.books.9780890425596.

12. Smart R, Pacula RL. Early evidence of the impact of cannabis legalization on cannabis use, cannabis use disorder, and the use of other substances: Findings from state policy evaluations. Am J Drug Alcohol Abuse. 2019;45(6):644-63. https://doi.org/10.1080/00952990.2019.1669626.

13. Wen H, Hockenberry JM, Cummings JR. The effect of medical marijuana laws on adolescent and adult use of marijuana, alcohol, and other substances. J Health Econ. 2015;42:64-80. https://doi.org/10. 1016/j.jhealeco.2015.03.007.

14. Lai HMX, Sitharthan T. Exploration of the comorbidity of cannabis use disorders and mental health disorders among inpatients presenting to all hospitals in New South Wales, Australia. Am J Drug Alcohol Abuse. 2012;38(6):567-74.

15. Agosti V, Nunes E, Levin F. Rates of psychiatric comorbidity among US residents with lifetime cannabis dependence. Am J Drug Alcohol Abuse. 2002;28(4):643-52. https://doi.org/10.1081/ADA-120015873.

16. Lai HMX, Huang QR. Comorbidity of mental disorders and alcohol- and drug-use disorders: Analysis of New South Wales inpatient data. Drug Alcohol Rev. 2009;28(3):235-42. https://doi.org/10.1111/j. 1465-3362.2008.00021.x.

17. Moitra E, Christopher PP, Anderson BJ, Stein MD. Coping-motivated marijuana use correlates with DSM-5 cannabis use disorder and psychological distress among emerging adults. Psychology of addictive behaviors: Journal of the Society of Psychologists in Addictive Behaviors. 2015;29(3):627-32. https://doi.org/10.1037/adb0000083.

18. Dorard G, Bungener C, Corcos M, Berthoz S. Self-esteem, coping, perceived social support and substance use in young adults with a cannabis dependence disorder. L'Encéphale: Revue de Psychiatrie Clinique Biologique et Thérapeutique. 2014;40(3):255-62. https://doi.org/10.1016/j.encep.2013.04. 014.

19. Dobkin PL, De Civita M, Paraherakis A, Gill K. The role of functional social support in treatment retention and outcomes among outpatient adult substance abusers. Addiction. 2002;97(3):347-56. https://doi.org/10.1046/j.1360-0443.2002.00083.x.

20. Warren CD, Fowler K, Speed D, Walsh A. The influence of social support on psychological distress in Canadian adults with bipolar disorder. Social Psychiatry and Psychiatric Epidemiology: The International Journal for Research in Social and Genetic Epidemiology and Mental Health Services. 2018;53(8):815-21. https://doi.org/10.1007/s00127-018-1529-7. 
21. Wareham S, Fowler K, Pike A. Determinants of depression severity and duration in Canadian adults: The moderating effects of gender and social support. J Appl Soc Psychol. 2007;37(12):2951-79. https://doi.org/10.1111/j.1559-1816.2007.00289.x.

22. Rapier R, McKernan S, Stauffer CS. An inverse relationship between perceived social support and substance use frequency in socially stigmatized populations. Addict Behav Rep. 2019;10. https://doi.org/ 10.1016/j.abrep.2019.100188.

23. van Rossum I, Boomsma M, Tenback D, Reed C, van Os J. Does cannabis use affect treatment outcome in bipolar disorder? A longitudinal analysis. J Nerv Ment Dis. 2009;197(1):35-40. https://doi. org/10.1097/NMD.0b013e31819292a6.

24. Gates P, Copeland J, Swift W, Martin G. Barriers and facilitators to cannabis treatment. Drug Alcohol Rev. 2012;31(3):311-9. https://doi.org/10.1111/j.1465-3362.2011.00313.x.

25. Statistics Canada. Canadian Community Health Survey (CCHS)-Mental Health. Ottawa: Statistics Canada; 2013.

26. WHO-CIDI. The world health organization world mental health composite international diagnostic interview. 2017. Retrieved from: https://www.hcp.med.harvard.edu/wmhcidi/.

27. Cuttler C, Mischley LK, Sexton M. Sex differences in cannabis use and effects: A cross-sectional survey of cannabis users. Cannabis Cannabinoid Res. 2016;1(1):166-75. https://doi.org/10.1089/can. 2016.0010.

28. Pacek LR, Mauro PM, Martins SS. Perceived risk of regular cannabis use in the United States from 2002 to 2012: differences by sex, age, and race/ethnicity. Drug Alcohol Depend. 2015;149:232-44. https://doi. org/10.1016/j.drugalcdep.2015.02.009.

29. Matheson J, Sproule B, Di Ciano P, et al. Sex differences in the acute effects of smoked cannabis: evidence from a human laboratory study of young adults. Psychopharmacology. 2020;237:305-16. https://doi.org/10.1007/s00213-019-05369-y.

30. Mauro PM, Carliner H, Brown QL, Hasin DS, Shmulewitz D, Rahim-Juwel R, Sarvet AL, Wall MM, Martins SS. Age differences in daily and nondaily cannabis use in the United States, 2002-2014. J Stud Alcohol Drugs. 2018;79(3):423-31.

31. Millar SR, Mongan D, O’Dwyer C, Long J, Smyth BP, Perry IJ, Galvin B. Correlates of patterns of cannabis use, abuse and dependence: evidence from two national surveys in Ireland. Eur J Public Health. 2021;31(2):441-7. https://doi.org/10.1093/eurpub/ckab007.

Publisher's Note Springer Nature remains neutral with regard to jurisdictional claims in published maps and institutional affiliations.

Tracy L. Gulliver is a Research Consultant with MQO Research. She specializes in applied research and evaluation techniques, including research design, advanced statistical analysis, and the analysis and interpretation of qualitative data. She has completed a Bachelor of Science (Honours) Degree in Psychology and a Master of Applied Psychological Science. Tracy received funding for her Master's research from the Social Sciences and Humanities Research Council of Canada, and completed the research under the supervision of Dr. Ken Fowler, PhD. Throughout her academic career, Tracy's research interests focused on social psychology and the impacts of social support on substance use disorder.

Dr. Ken Fowler was appointed Director of Memorial University’s Student Wellness and Counselling Centre on August 1st, 2020. Prior to this, he spent 20 years as a faculty member with the Department of Psychology, serving as Department Head for the most recent 2 years. Dr. Fowler lectures in health and social psychology, supervises the thesis work of Honours, Masters, PhD, and PsyD students, and conducts research focusing on social context predictors and consequences of various mental health issues including bipolar disorder, PTSD, depression, ADHD/ADD, and anxiety disorder. 


\section{Authors and Affiliations}

Tracy L. Gulliver ${ }^{1}(\mathbb{D}) \cdot$ Ken Fowler $^{2}$ (D)

Ken Fowler

kenfowler@mun.ca

1 Department of Psychology, Memorial University of Newfoundland, St. John's, NL A1B 3V6, Canada

2 Department of Psychology, Memorial University of Newfoundland, St. John's, NL A1B 3X9, Canada 